\title{
Smooth muscle-specific HuR knockout induces defective autophagy and atherosclerosis
}

\author{
Shanshan Liu' ${ }^{1,2}$, Xiuxin Jiang ${ }^{3}$, Xiuru Cui ${ }^{1}$, Jingjing Wang ${ }^{4}$, Shangming Liu ${ }^{5}$, Hongxuan Li ${ }^{1}$, Jianmin Yang ${ }^{1}$, \\ Cheng Zhang ${ }^{1}$ and Wencheng Zhang $\mathbb{D}^{1,2}$
}

\begin{abstract}
Human antigen R (HuR) is a widespread RNA-binding protein involved in homeostatic regulation and pathological processes in many diseases. Atherosclerosis is the leading cause of cardiovascular disease and acute cardiovascular events. However, the role of HuR in atherosclerosis remains unknown. In this study, mice with smooth muscle-specific HuR knockout ( $\mathrm{HuR}^{\mathrm{SMKO}}$ ) were generated to investigate the role of HuR in atherosclerosis. HuR expression was reduced in atherosclerotic plaques. As compared with controls, $\operatorname{HuR}^{\text {SMKO }}$ mice showed increased plaque burden in the atherosclerotic model. Mechanically, HuR could bind to the mRNAs of adenosine 5'-monophosphate-activated protein kinase (AMPK) a1 and AMPKa2, thus increasing their stability and translation. HuR deficiency reduced p-AMPK and LC3II levels and increased p62 level, thereby resulting in defective autophagy. Finally, pharmacological AMPK activation induced autophagy and suppressed atherosclerosis in $\mathrm{HuR}^{\mathrm{SMKO}}$ mice. Our findings suggest that smooth muscle HuR has a protective effect against atherosclerosis by increasing AMPK-mediated autophagy.
\end{abstract}

\section{Introduction}

Atherosclerosis is a chronic and systemic vascular inflammatory process, the pathological basis of coronary artery disease, myocardial infarction, and stroke ${ }^{1}$. Despite many treatment options, it remains the leading cause of death worldwide. Developing new strategies to prevent plaque formation and rupture has become an important research area. Atherosclerosis is initiated by endothelial dysfunction and vascular inflammation caused by cardiovascular risk factors such as hyperlipidemia and hypertension. Lipoproteins in the blood enter the arterial wall from the damaged endothelial cells ${ }^{2,3}$. Inflammatory factors can stimulate monocytes and vascular smooth muscle

\footnotetext{
Correspondence: Wencheng Zhang (zhangwencheng@sdu.edu.cn)

${ }^{1}$ The Key Laboratory of Cardiovascular Remodeling and Function Research, Chinese Ministry of Education, Chinese National Health Commission and Chinese Academy of Medical Sciences, The State and Shandong Province Joint Key Laboratory of Translational Cardiovascular Medicine, Department of Cardiology, Qilu Hospital, Cheeloo College of Medicine, Shandong University, Jinan, China

${ }^{2}$ Cardiovascular Disease Research Center of Shandong First Medical University, Central Hospital Affiliated to Shandong First Medical University, Jinan, China Full list of author information is available at the end of the article Edited by G.M. Fimia
}

cells (VSMCs) to engulf oxidized low-density lipoprotein (ox-LDL) and form foam cells ${ }^{4}$. Disordered lipid metabolism, inflammation, and endothelial injury all seem to play major roles in atherosclerosis ${ }^{4,5}$. However, the pathogenesis of atherosclerosis is still unclear and needs further study.

VSMCs are crucial in atherosclerosis. Most foam cells in early atherosclerosis are derived from VSMCs ${ }^{6}$. Aberrant proliferation and migration followed by phenotypic switching of VSMCs are involved in the formation of atherosclerosis ${ }^{7}$. VSMCs produce the extracellular matrix, which forms the fibrous cap to prevent plaque rupture ${ }^{8}$. The death and senescence of VSMCs participate in the formation of atherosclerotic plaque and also promote the instability of plaque in advanced lesions ${ }^{7,9}$. However, the specific regulatory mechanism of VSMCs in atherosclerosis is not clear.

Autophagy is an essential subcellular process that has a "housekeeping" role in the normal physiological functions of the body. Autophagy participates in numerous physiological and pathological processes, including cell differentiation, growth regulation, aging, immunity, and

\section{(c) The Author(s) 2021}

(c) (i) Open Access This article is licensed under a Creative Commons Attribution 4.0 International License, which permits use, sharing, adaptation, distribution and reproduction cc) in any medium or format, as long as you give appropriate credit to the original author(s) and the source, provide a link to the Creative Commons license, and indicate if changes were made. The images or other third party material in this article are included in the article's Creative Commons license, unless indicated otherwise in a credit line to the material. If material is not included in the article's Creative Commons license and your intended use is not permitted by statutory regulation or exceeds the permitted use, you will need to obtain permission directly from the copyright holder. To view a copy of this license, visit http://creativecommons.org/licenses/by/4.0/. 
tumor suppression ${ }^{10}$. Autophagy is a multi-step process that requires a variety of autophagy-related proteins that take part in nucleation, expansion, and finally fusion with lysosomes of autophagosomes ${ }^{11,12}$. Accumulating evidence suggests that autophagy is involved in the occurrence and development of atherosclerosis and related diseases $^{13,14}$. Smooth muscle-specific Atg7 knockout can promote atherosclerosis ${ }^{15}$, but the molecular mechanism of autophagy in atherosclerosis still needs elucidation.

Human antigen $\mathrm{R}(\mathrm{HuR})$ is a ubiquitous and conserved RNA-binding protein that binds to AU-rich elements (AREs) and alters ARE-mediated mRNA turnover and translation ${ }^{16}$. HuR binds to an extensive list of RNAs that participate in cell proliferation, apoptosis, and differentiation ${ }^{17,18}$. Therefore, HuR may have an important effect on both pathologic and physiologic functions. Although cancer is the most widely studied disease associated with $\mathrm{HuR}^{19}$, the molecule is also reported to take part in chronic inflammation and nervous system diseases ${ }^{18,20}$. Our recent work showed that adipose tissuespecific HuR knockout caused obesity and metabolic disorders $^{21}$. Also, knockout of HuR enhanced VSMC contraction and hypertension ${ }^{22}$. However, the role of HuR in autophagy and atherosclerosis remains unclear.

In this study, we examined HuR expression in atherosclerotic plaques and found its expression decreased. Using smooth muscle-specific HuR knockout $\left(\mathrm{HuR}^{\mathrm{SMKO}}\right)$ mice, smooth-muscle HuR deletion promoted atherosclerosis by inducing defective autophagy.

\section{Results}

\section{HuR levels were reduced in atherosclerotic plaque}

To investigate the role of HuR in atherosclerosis, we fed $\mathrm{ApoE}^{-1-}$ mice with an ND or HFD for 12 weeks. HuR protein level was decreased in aortas from the HFD group (Fig. 1A, B). As compared with the ND group, for the HFD group, HuR was downregulated in atherosclerotic lesions (Fig. 1C). As well, HuR mRNA level was significantly reduced in aortas from the HFD group (Fig. 1D). HuR protein level was decreased in VSMCs induced by ox-LDL at 24, 48, 72, and $96 \mathrm{~h}$ (Fig. 1E, F). For further study, we used $\mathrm{HuR}^{\mathrm{SMKO}}$ mice generated from a hybrid of HuR-floxed mice and $\alpha$-SMA-Cre transgenic mice. HuR protein level was downregulated in aortas from $\mathrm{HuR}^{\mathrm{SMKO}}$ mice (Fig. 1G, H). HuR was not expressed in the aortic smooth muscle layer of $\mathrm{HuR}^{\mathrm{SMKO}}$ mice as compared with control mice (Fig. 1I). In summary, the expression of HuR in atherosclerotic plaques was decreased, which suggests that $\mathrm{HuR}$ acts on atherosclerosis.

\section{HuR deletion in smooth muscle exacerbated atherosclerosis}

To explore the role of smooth-muscle HuR in atherosclerosis, control, and $\mathrm{HuR}^{\mathrm{SMKO}}$ mice were injected with
rAAV/D377Y-mPCSK9 then fed a Paigen diet for 12 weeks. The proportion of atherosclerotic surface lesions was greater in $\mathrm{HuR}^{\mathrm{SMKO}}$ than control mice $(33.98 \pm 6.56 \%$ vs $14.68 \pm 2.47 \%, p<0.001)$ (Fig. 2 A). Oilred O-stained aortic roots showed significantly increased lesion area in $\mathrm{HuR}^{\mathrm{SMKO}}$ than control mice $(43.38 \pm 2.75 \%$ vs $28.80 \pm 4.66 \%, p<0.01$ ) (Fig. $2 \mathrm{~B}$ ). Furthermore, deletion of HuR increased macrophage accumulation (Fig. 2C) and matrix metalloproteinase 2 (MMP2) level (Supplementary Fig. 2B), decreased collagen content (Fig. 2D). However, VSMC content and total monocyte/macrophages did not differ from controls (Fig. 2E and Supplementary Fig. 2A). From the above results, we calculated the plaque vulnerability index, which was elevated after HuR deficiency (Fig. 2F). Taken together, lack of HuR in VSMCs promoted the development of atherosclerosis.

\section{Loss of HuR promoted apoptosis in atherosclerosis}

Numerous studies have confirmed the existence of apoptosis in atherosclerotic plaques, which even affects the stability of plaques ${ }^{7,23}$. To determine whether $\mathrm{HuR}$ knockout affected apoptosis, aortic root sections underwent TUNEL staining. Loss of HuR markedly increased the TUNEL-positive SMCs in $\mathrm{HuR}^{\mathrm{SMKO}}$ versus control mice (Fig. 3A). Meanwhile, the expression of the apoptosis-related protein cleaved caspase- 3 was upregulated in aortic roots of $\mathrm{HuR}^{\mathrm{SMKO}}$ mice (Fig. $3 \mathrm{~B}$ ). Besides, HuR deletion also increased the level of cleaved caspase- 3 in SMCs (Fig. 3D). Also, the serum levels of TC, TG, LDL$\mathrm{C}$ were higher in $\mathrm{HuR}^{\mathrm{SMKO}}$ than control mice (Fig. 3C). To learn why $\mathrm{HuR}^{\mathrm{SMKO}}$ mice have the phenotype of dyslipidemia, HuR expression in hepatocytes and liver fibroblasts were detected. Results showed that $\mathrm{HuR}$ expression was no difference in hepatocytes, but decreased in liver fibroblasts from $\mathrm{HuR}^{\mathrm{SMKO}}$ mice compared with control (Supplementary Fig. 1A, B). We further examined serum aspartate transaminase (AST) and alanine transaminase (ALT) levels and found elevated AST level from $\mathrm{HuR}^{\mathrm{SMKO}}$ mice (Supplementary Fig. 1C, D). Thus, HuR deficiency in smooth muscle increased apoptosis in atherosclerotic mice, and loss of HuR in liver fibroblasts may contribute to hepatic dysfunction and dyslipidemia.

\section{HuR deletion resulted in defective autophagy}

VSMC autophagy was reported to be important during the process of atherosclerosis ${ }^{14,15}$. To detect whether HuR could regulate autophagy, we used transmission electron microscopy after VSMCs were infected with ad-HuR or ad-LacZ for $48 \mathrm{~h}$. HuR overexpression increased the number of autophagosomes (Fig. 4A). Also, autophagic flux was monitored in VSMCs by infection with adGFP-mRFP-LC3II. As compared with controls, VSMCs with $\mathrm{HuR}$ overexpression by ad-HuR infection showed 

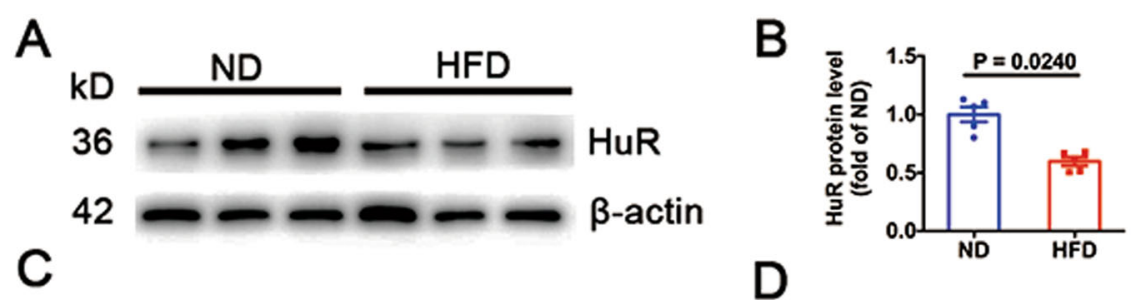

ND HFD
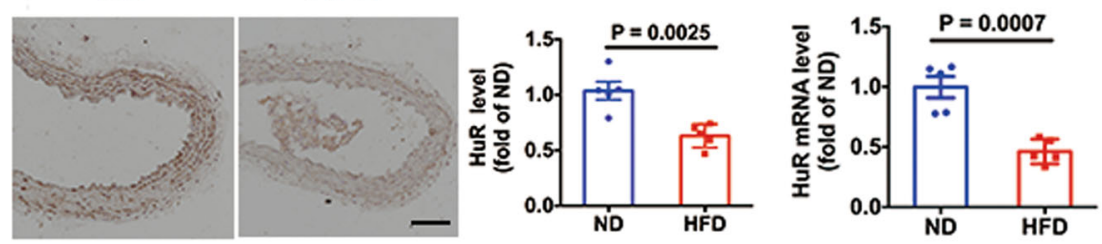

E
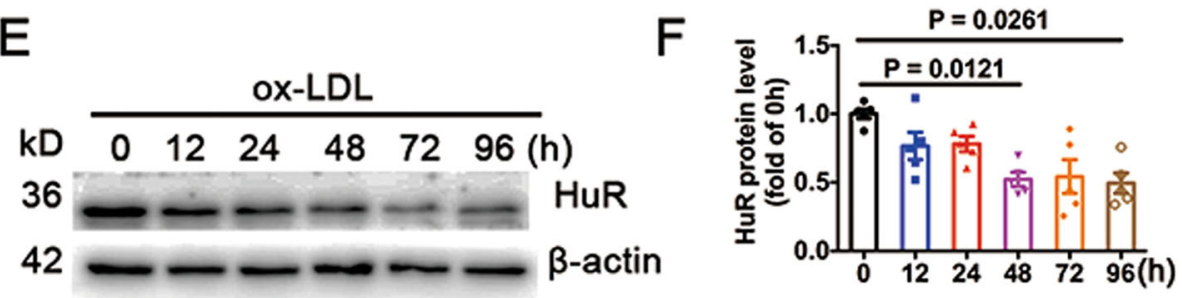

G

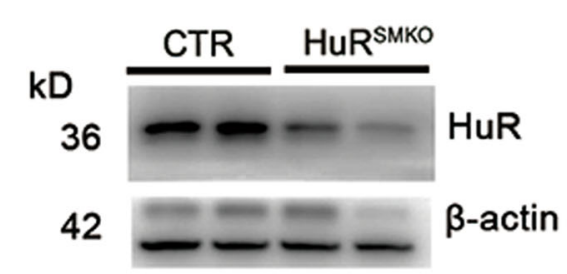

HuR

a-SMA
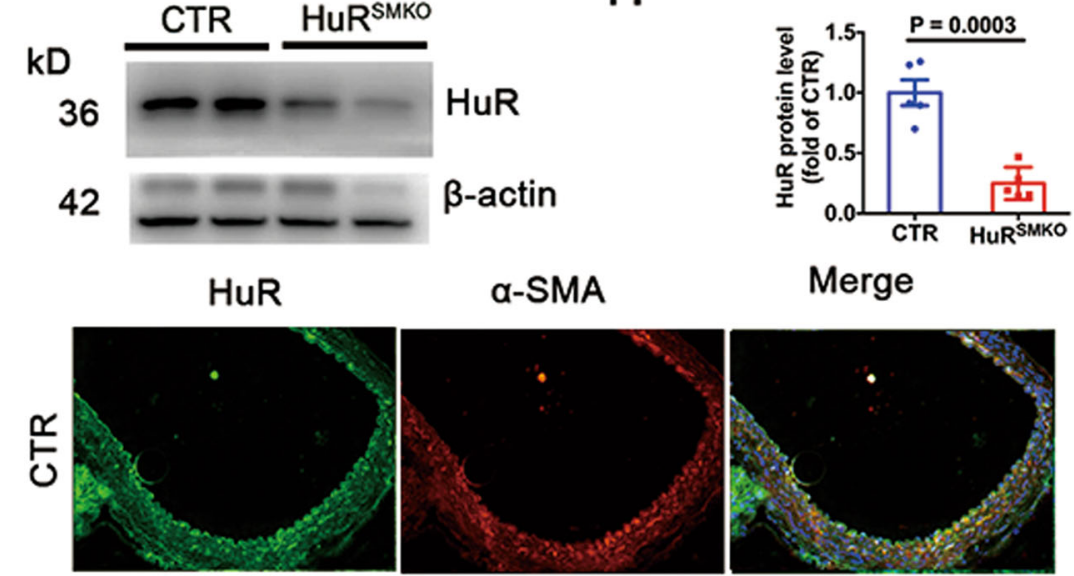

Merge
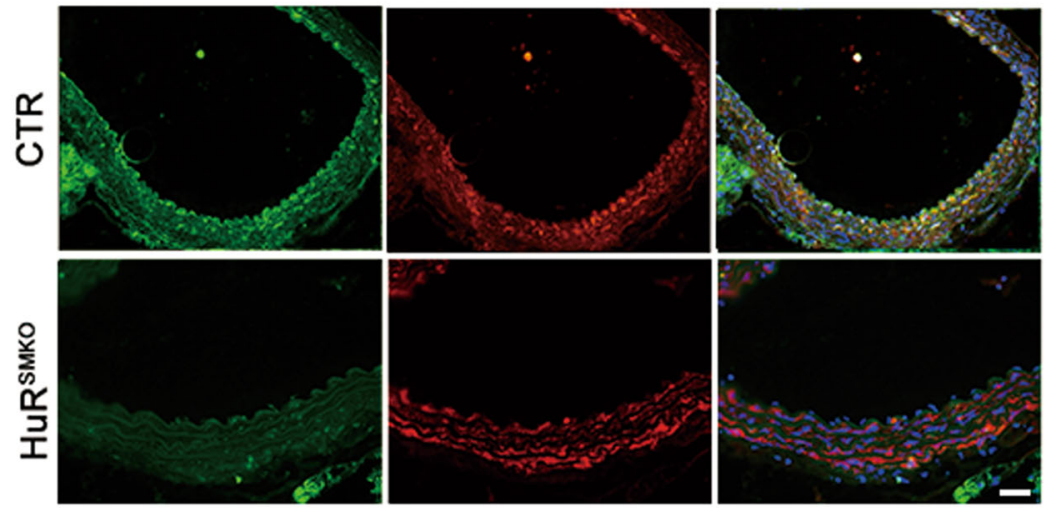

Fig. 1 HuR levels were reduced in atherosclerotic plaque. A, B Western blot analysis of HuR protein level in aortas from apolipoprotein Eknockout $\left(\mathrm{ApoE}^{-/-}\right.$) mice fed a high-fat diet (HFD) or normal diet (ND) $(n=5)$. $\mathbf{C}$ Immunohistochemical staining of HuR in aortas from ApoE ${ }^{-/-}$mice fed an HFD or ND $(n=5)$. D Quantitative RT-PCR analysis of HuR mRNA level in aortas from ApoE ${ }^{-/-}$mice fed an HFD or ND ( $\left.n=5\right)$. E, F Western blot analysis of HuR protein level in vascular smooth muscle cells (VSMCs) induced by $50 \mu \mathrm{g} / \mathrm{ml}$ oxidized low-density lipoprotein (ox-LDL) at different times $(n=5)$. G, H Western blot analysis of HuR protein level in aortas from control and HuR ${ }^{\text {SMKO }}$ mice $(n=5)$. I Immunofluorescent staining of aortas from control and HuR ${ }^{\text {SMKO }}$ mice to determine HuR (green) and a-SMA (a-smooth muscle actin; red) localization. Scale bar = $50 \mu m$. 

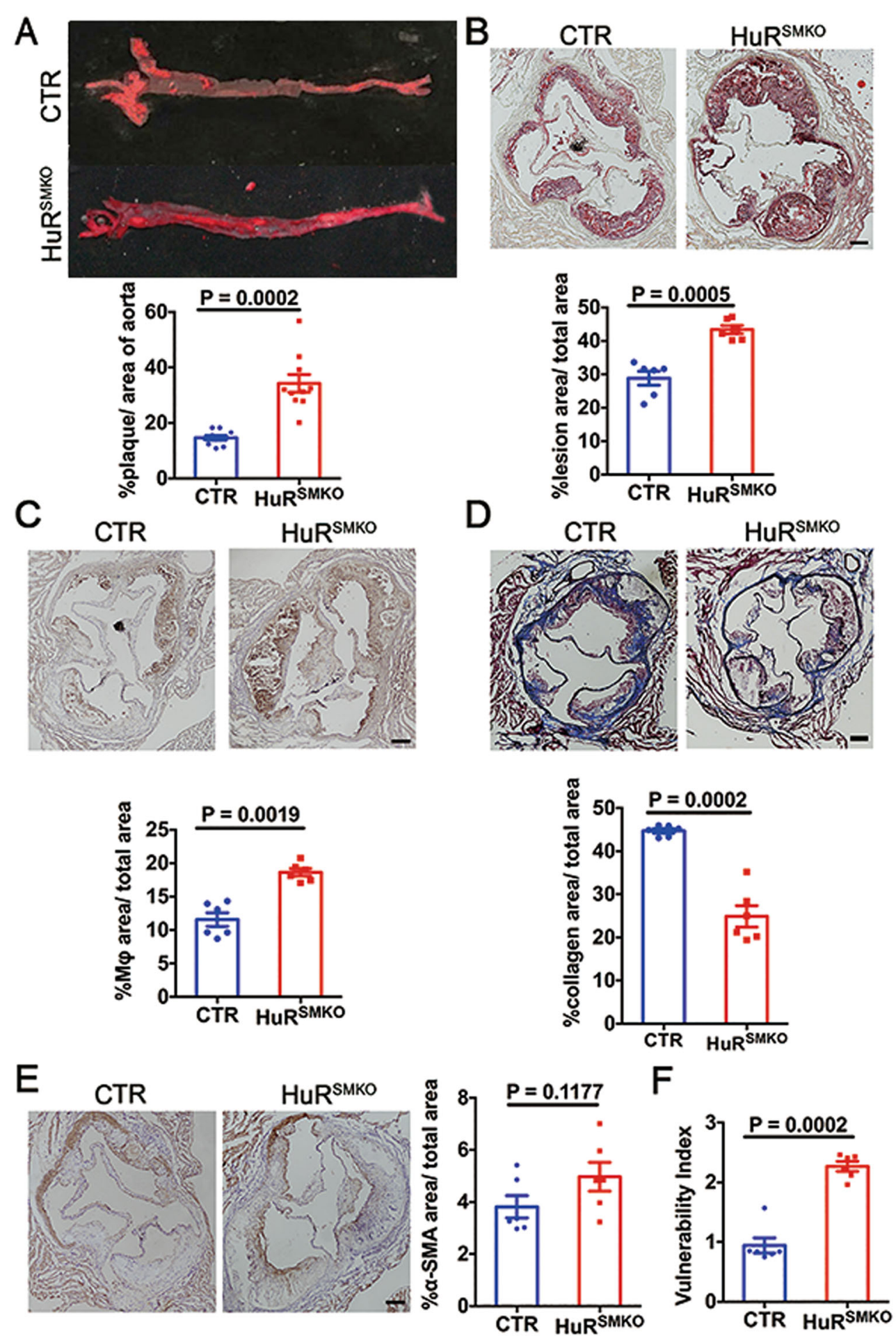

Fig. 2 HuR deletion in smooth muscle cells exacerbated atherosclerosis. CTR and HuR ${ }^{\text {SMKO }}$ mice were injected with rAAV/D377Y-mPCSK9 and fed a Paigen diet for 12 weeks. A Oil-red O staining in aortas $(n=10)$. B Oil-red O staining in aortic roots $(n=6)$. Scale bar $=200 \mu \mathrm{m}$.

c Immunohistochemical staining of MOMA-2 in aortic roots $(n=6)$. Scale bar $=200 \mu \mathrm{m}$. D Masson staining in aortic roots $(n=6)$. Scale bar $=200 \mu \mathrm{m}$. E Immunohistochemical staining of a-SMA $(n=6)$. Scale bar $=200 \mu \mathrm{m}$. F Vulnerable index of atherosclerotic plaques $(n=6)$. 

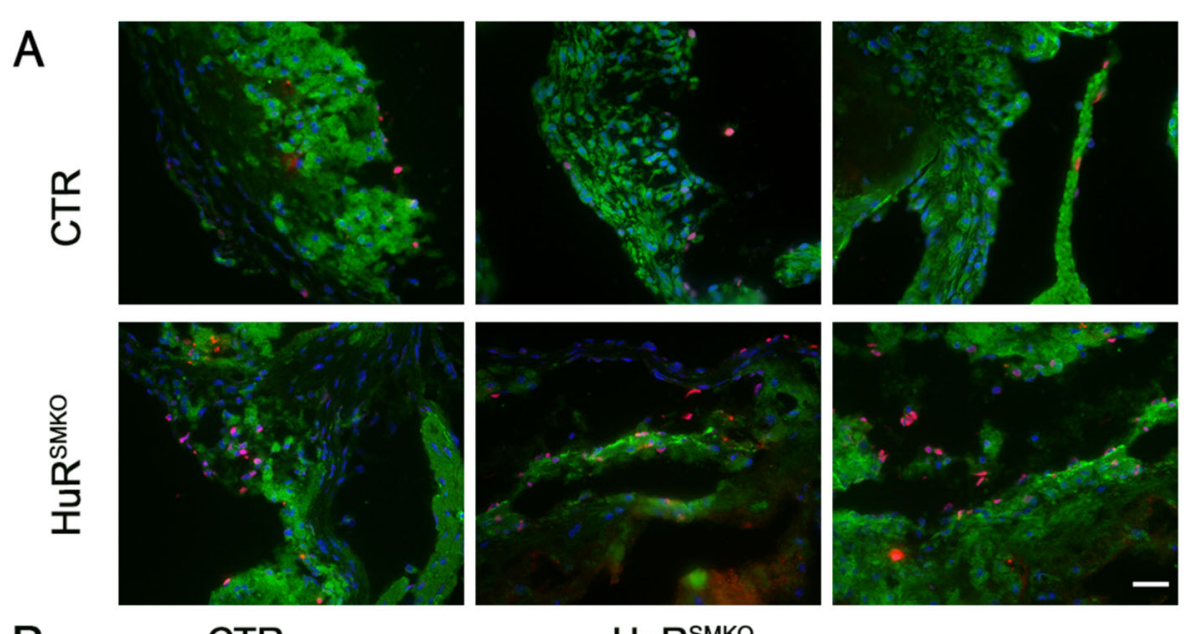

B
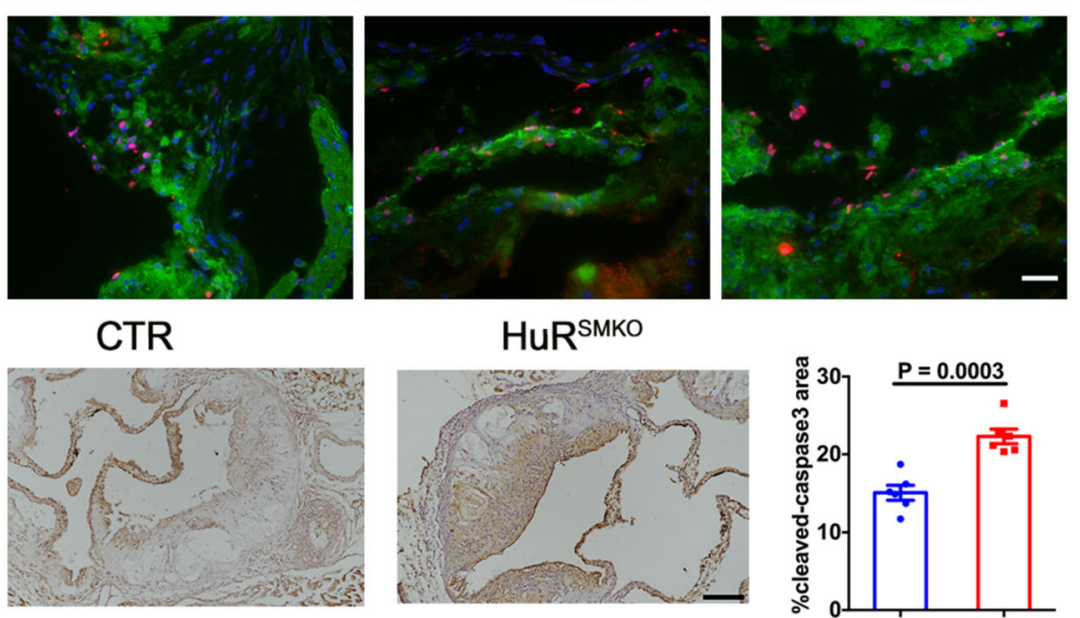

HuR ${ }^{\text {SMKO }}$
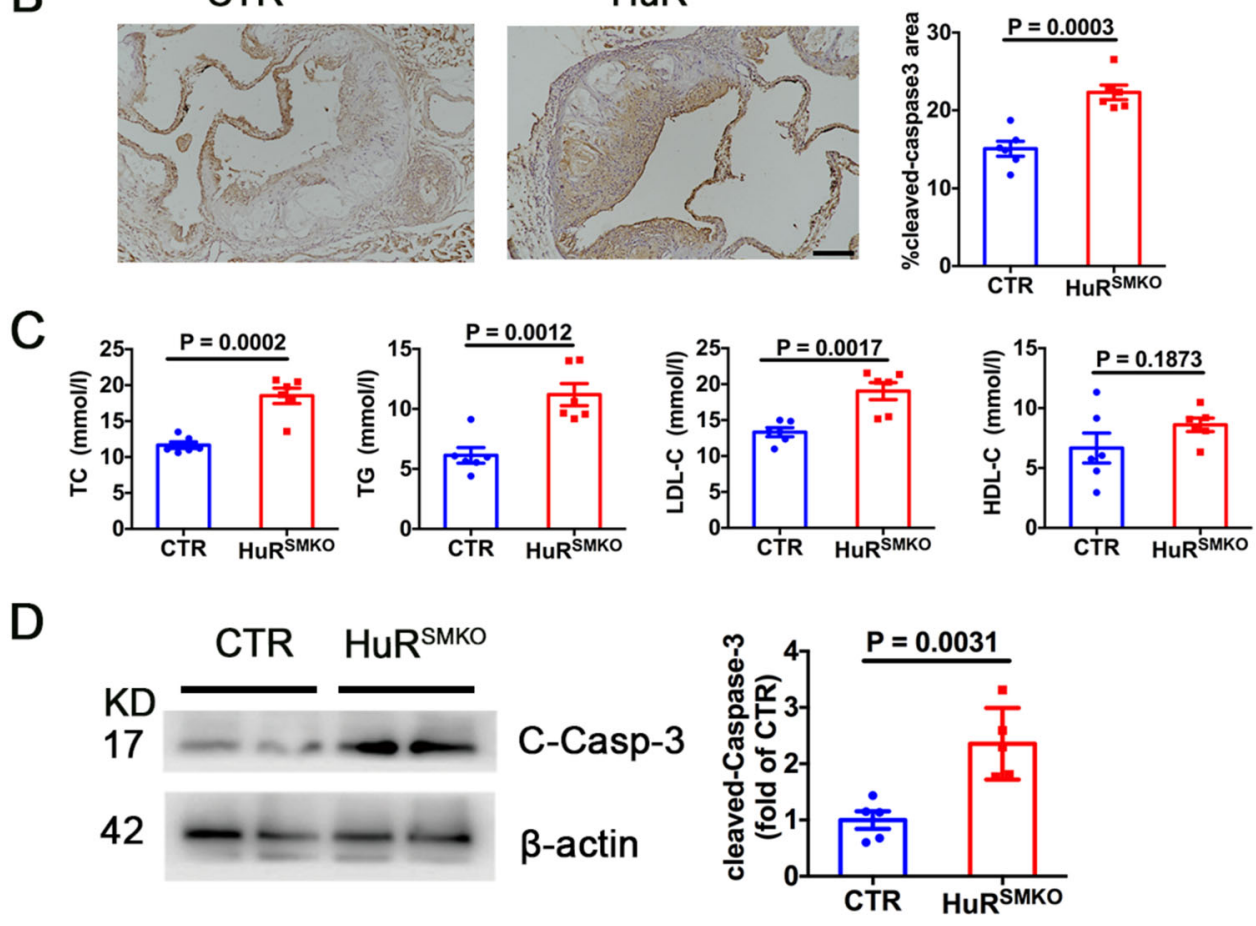

Fig. 3 Loss of HuR promoted apoptosis in atherosclerosis. A Immunofluorescent staining of aortic roots from CTR and HuR ${ }^{\text {SMKO }}$ mice to determine TUNEL-positive VSMCs. Red puncta denotes TUNEL-positive cells. Green region denotes a-SMA. Scale bar $=20 \mu \mathrm{m}$. B Immunohistochemical staining of cleaved caspase-3 in aortic roots $(n=6)$. Scale bar $=50 \mu \mathrm{m}$. C Serum lipid profiles (total cholesterol [TC], triglycerides [TG], high-density lipoprotein cholesterol [HDL-C], and low-density lipoprotein cholesterol [LDL-C]) $(n=6)$. D Western blot analysis of cleaved caspase-3 in control and HuR-deficient SMCs $(n=5)$.

an increased number of $\mathrm{GFP}^{+} / \mathrm{RFP}^{+}$and $\mathrm{GFP}^{-} / \mathrm{RFP}^{+}$ LC3II puncta (Fig. 4B-D). However, VSMCs knocked down by HuR siRNA transfection showed decreased number of $\mathrm{GFP}^{+} / \mathrm{RFP}^{+}$and $\mathrm{GFP}^{-} / \mathrm{RFP}^{+}$LC3II puncta as compared with controls (Fig. 4E-G). Thus, HuR overexpression induced autophagic flux, and loss of HuR suppressed autophagic flux and resulted in defective autophagy.

\section{AMPKa1 and AMPKa2 were the target genes of HuR}

As a key factor in cellular energy metabolism and activator of autophagy, AMP-activated protein kinase (AMPK) participates in various physiological and pathological processes ${ }^{24}$. The mRNA levels of AMPK $\alpha 1$ and $A M P K \alpha 2$ were decreased in aortas from $\mathrm{HuR}^{\mathrm{SMKO}}$ mice (Fig. 5A). To detect whether AMPK $\alpha$ was the HuR target gene, the sequence of mouse AMPK $\alpha$ transcripts was 

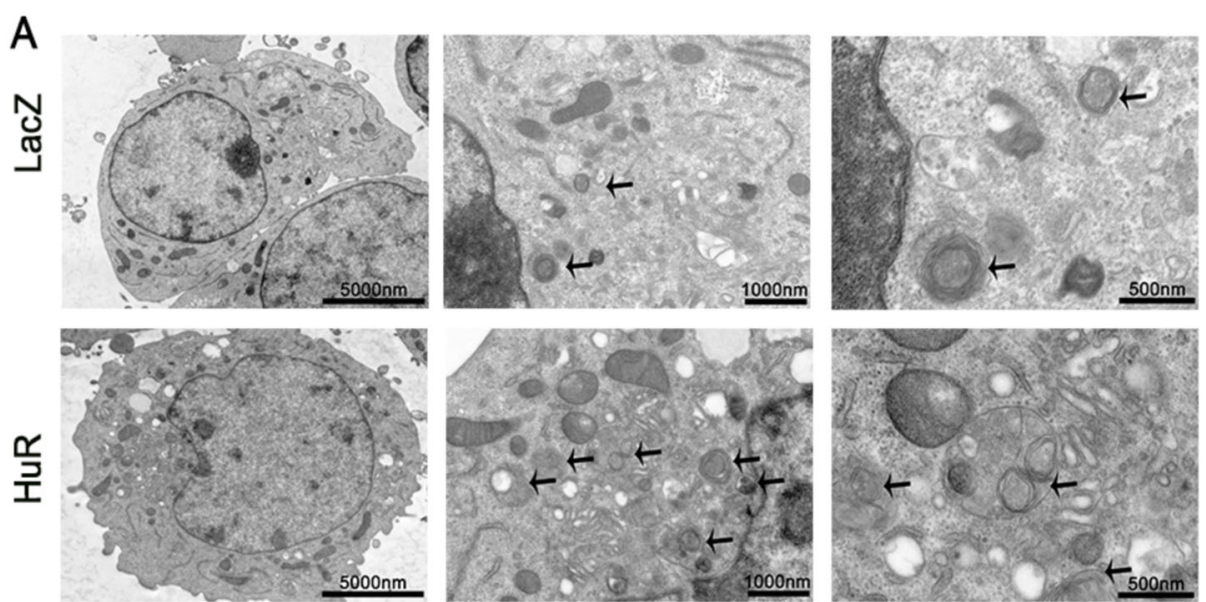

B
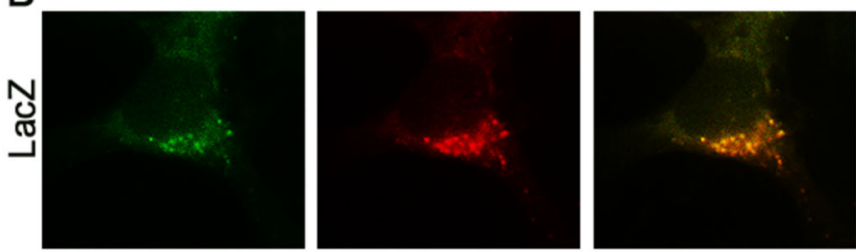

C
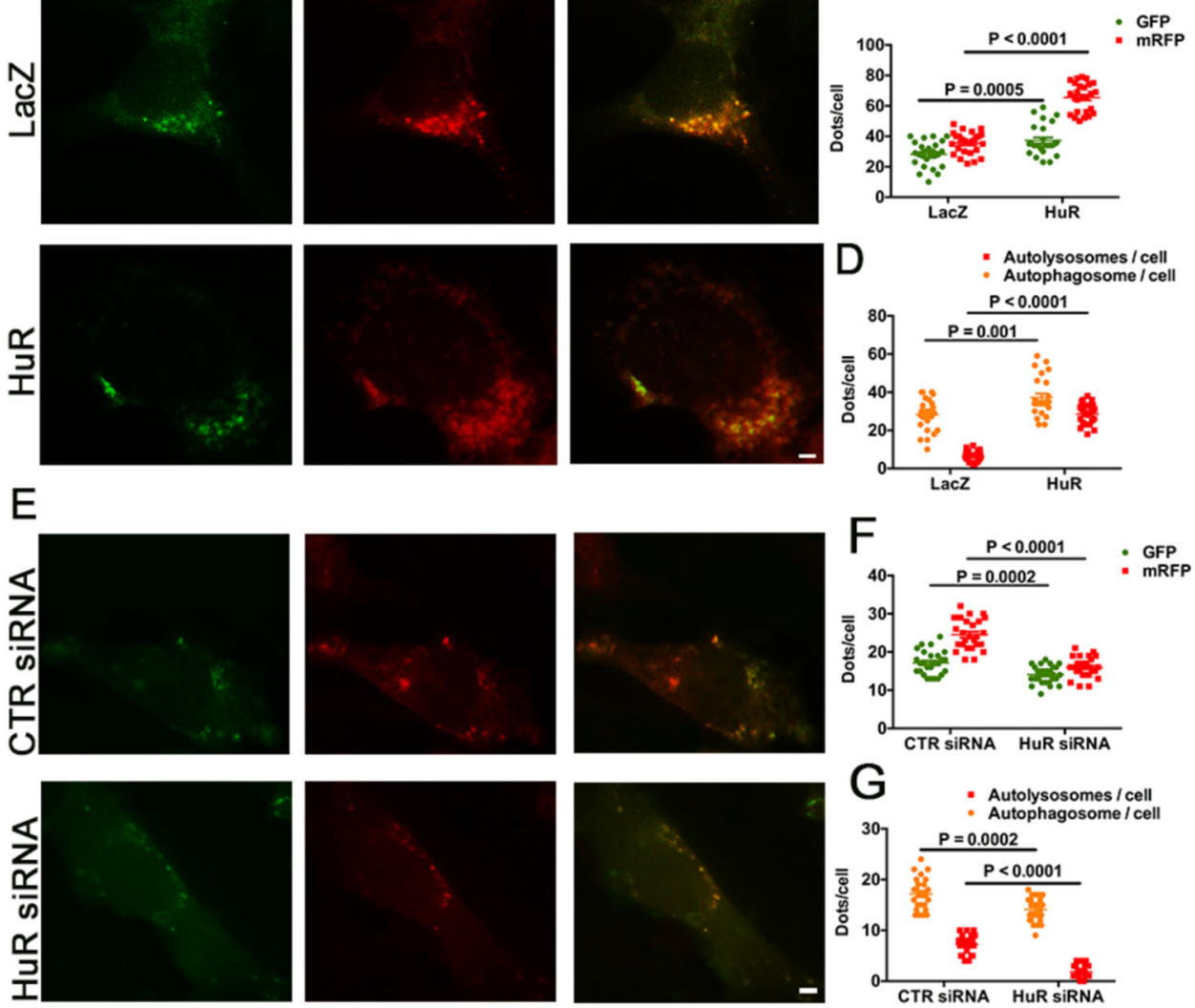

Fig. 4 HuR deletion resulted in defective autophagy. A Transmission electron microscopy of autophagosomes (black arrow) in VSMCs infected with ad-HuR or ad-LacZ. Scale bar $=5000 \mathrm{~nm}$ (left), $1000 \mathrm{~nm}$ (middle), $500 \mathrm{~nm}$ (right). B-D fluorescence photomicrographs (B) and quantification (C, D) of GFP-mRFP-LC3II puncta in VSMCs infected with ad-HuR or ad-LacZ $(n=26)$. Scale bar $=10 \mu \mathrm{m}$. E-G Fluorescence photomicrographs $(\mathbf{E})$ and quantification $(\mathbf{F}, \mathbf{G})$ of GFP-mRFP-LC3II puncta in VSMCs transfected with control or HuR siRNA $(n=26)$. Scale bar $=10 \mu m$. Yellow puncta denotes autophagosome. Red puncta denotes autolysosome.

analyzed and there are 3 AREs in the $3^{\prime}$ UTR of AMPK $\alpha 1$ and 4 AREs in the $3^{\prime}$ UTR of AMPK 2 . Next, we examined the binding of HuR to AMPK $\alpha 1$ and AMPK $\alpha 2$ mRNA by RNA immunoprecipitation and mRNA stability assay. HuR could bind to the mRNAs of AMPK $\alpha 1$ and
AMPK $\alpha 2$ (Fig. $5 B$ ). Meanwhile, the stability of AMPK $\alpha 1$ and AMPK 2 mRNAs was increased by HuR overexpression (Fig. 5C, D), but decreased by HuR deficiency (Supplementary Fig. 3A, B). Then we further verified the relationship between $\mathrm{HuR}$ and AMPK $\alpha$. VSMCs were 


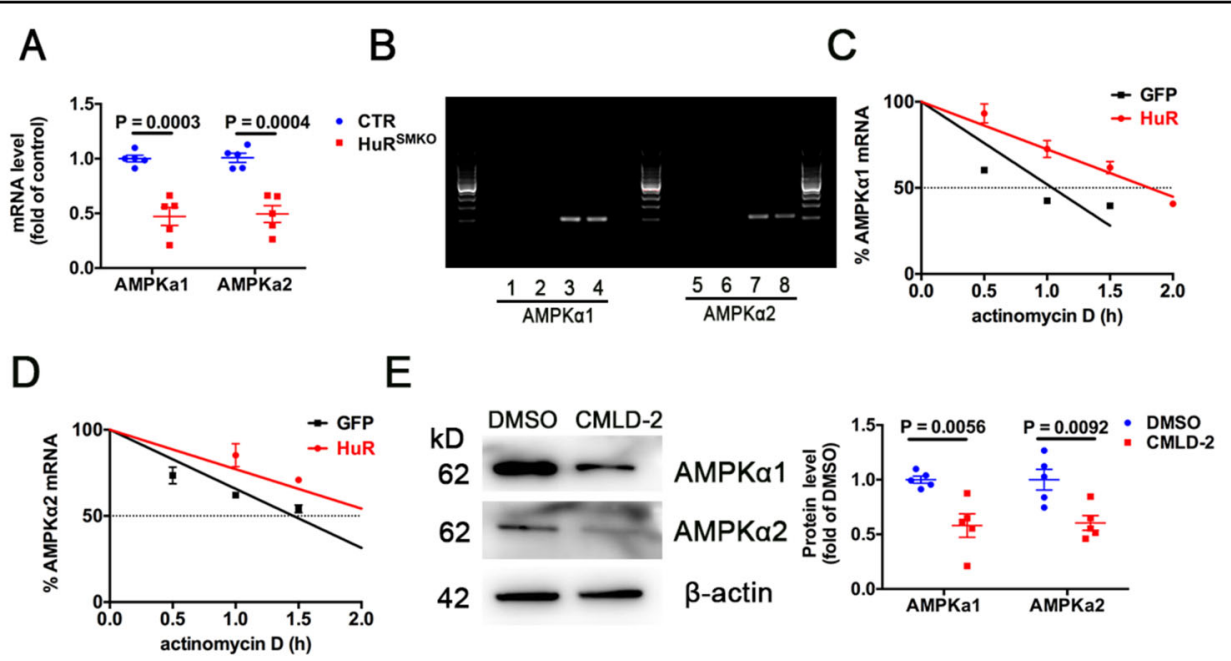

F

G

$\mathrm{H}$

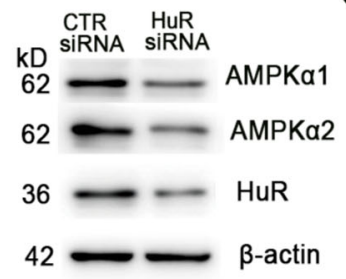

kD GFP HuR

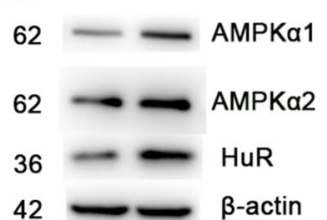

kD PBS HuR

$\mathrm{kD}$
$62=-\mathrm{AMPK \alpha 1}$

62

AMPKa2

42

$\beta$-actin

HuR

42

$\beta$-actin

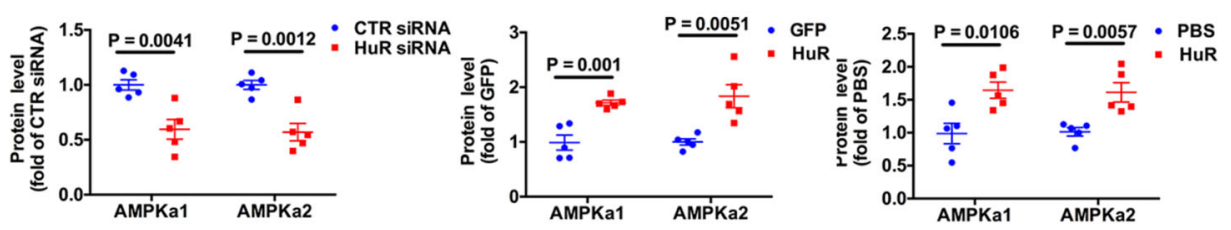

I

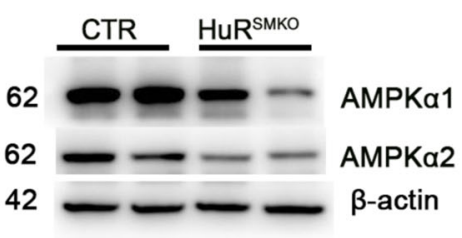

\section{AMPKa1 AMPKa2}
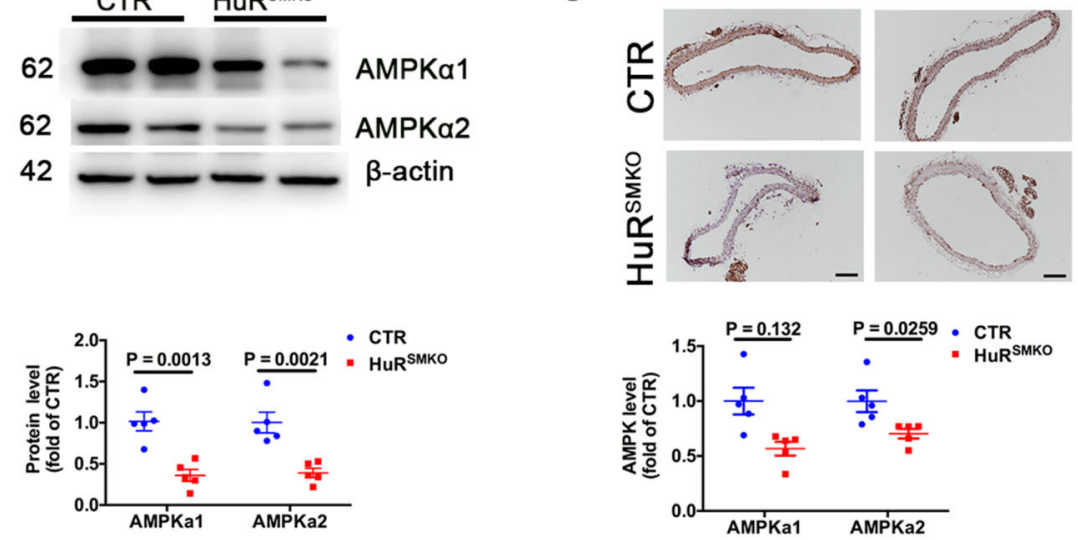

Fig. 5 AMPKa1 and AMPKa2 are the target genes of HuR. A Quantitative RT-PCR analysis of aortic mRNA levels of AMPKa1, AMPKa2 from CTR and $\mathrm{HuR}^{\text {SMKO }}$ mice $(n=5)$. B RNA immunoprecipitation with anti-HuR or control lgG antibody. Lanes 1, 5, no template PCR control; lanes 2, 6, IgG RNA immunoprecipitation; lanes 3, 7, anti-HuR RNA immunoprecipitation; lanes 4, 8, 10\% input. C, D VSMCs were infected with adenovirus-expressing GFP (green fluorescent protein) or HuR and then treated with actinomycin D $(5 \mu \mathrm{g} / \mathrm{ml})$. Quantified RT-PCR analysis of percentage mRNA levels of AMPKa1 (C) and AMPKa2 (D) $(n=5)$ in VSMCs. E Western blot analysis of AMPKa1 and AMPKa2 in VSMCs treated with DMSO or $30 \mu M$ CMLD-2 for $24 \mathrm{~h}(n=5)$. $\mathbf{F}$ Western blot analysis of AMPKa1 and AMPKa2 in VSMCs transfected with CTR siRNA or HuR siRNA for $48 \mathrm{~h}(n=5)$. Western blot analysis of AMPKa1 and AMPKa2 in VSMCs, G infected with ad-GFP or HuR $(n=5)$, and $\mathbf{H}$ treated with PBS or $0.5 \mu \mathrm{g} / \mu$ recombinant HuR protein for $48 \mathrm{~h}(n=5)$. I Western blot analysis of AMPKa1 and AMPKa2 in aortas from CTR and HuR ${ }^{\text {SMKO }}$ mice $(n=5)$. J Immunohistochemical staining of AMPKa1 and AMPKa2 in aortas from CTR and HuR ${ }^{\text {SMKO }}$ mice $(n=5)$. Scale bar $=100 \mu \mathrm{m}$. 
stimulated with $30 \mu \mathrm{M}$ CMLD-2, a HuR inhibitor, for $24 \mathrm{~h}$. The protein expression of AMPKa was sharply reduced in VSMCs with CMLD-2 treatment (Fig. 5E) and AMPK $\alpha 1$ and AMPK $\alpha 2$ protein levels in VSMCs were reduced after HuR knockdown by HuR siRNA transfection (Fig. 5F). In contrast, the levels of AMPK $\alpha 1$ and AMPK $\alpha 2$ were increased in VSMCs with HuR overexpression by ad-HuR infection or HuR recombinantprotein stimulation (Fig. 5G, H). The protein levels of AMPK $\alpha 1$ and AMPK $\alpha 2$ were significantly reduced in $\mathrm{HuR}^{\mathrm{SMKO}}$ versus control mice (Fig. 5I). Immunohistochemical staining results for AMPK $\alpha 1$ and AMPK $\alpha 2$ in aortas from control and $\mathrm{HuR}^{\mathrm{SMKO}}$ mice were consistent with the above results (Fig. 5J). The effect of HuR on AMPK expression was further confirmed by oxLDLstimulated SMCs after HuR knockdown or expression (Supplementary Fig. 4A, B). Therefore, AMPK $\alpha 1$ and AMPK $\alpha 2$ are the target genes of $\mathrm{HuR}$.

\section{HuR positively regulates autophagy}

Because AMPK could induce autophagy ${ }^{25}$ and AMPKa is the $\mathrm{HuR}$ target gene, we investigated whether $\mathrm{HuR}$ regulates autophagy. HuR inhibition with CMLD-2 or HuR siRNA decreased levels of p-AMPK and LC3II and increased p62 level in VSMCs (Fig. 6A, B). In contrast, levels of p-AMPK and LC3II were elevated and that of p62 was decreased with HuR overexpression by ad-HuR infection or HuR recombinant-protein stimulation (Fig. 6C, D). Furthermore, as compared with control mice, $\mathrm{HuR}^{\mathrm{SMKO}}$ mice showed lower levels of p-AMPK and LC3II and higher level of p62 (Fig. 6E). Thus, HuR positively regulates autophagy.

\section{Pharmacological AMPK activation induced autophagy and suppressed atherosclerosis in $\mathrm{HuR}^{\mathrm{SMKO}}$ mice}

To further demonstrate that HuR regulates autophagy via AMPK, VSMCs were transfected with control or $\mathrm{HuR}$ siRNA then treated with the AMPK activator A769662. The levels of p-AMPK and LC3II were increased with A769662 in control and HuR-deficient VSMCs (Fig. 7A). In animal experiments, control and $\mathrm{HuR}^{\mathrm{SMKO}}$ mice were given an intraperitoneal injection of A769662 daily and fed a Paigen diet for 12 weeks after single intravenous injection of rAAV/D377Y-mPCSK9. The plaque area was significantly decreased after A769662 treatment in control and $\mathrm{HuR}^{\mathrm{SMKO}}$ mice (Fig. 7B, C). Also, A769662 reduced the number of apoptotic cells (Fig. 7D). Furthermore, only TC and LDL-C blood levels were slightly reduced with A769662 treatment (Fig. 7E). Thus, smooth-muscle HuR protects against the development of atherosclerosis via AMPK-mediated autophagy.

\section{Discussion}

In this study, the expression of HuR was decreased in atherosclerotic plaques from $\mathrm{ApoE}^{-/-}$mice with an HFD for 12 weeks. However, Rudolf Pullmann and Mitali Ray reported that increased HuR expression in atherosclerotic plaques from patients with neointimal proliferation and $\mathrm{LDLR}^{-1-}$ mice lacking of IL-19 ${ }^{26,27}$. These results suggested that HuR may play an important and complicated role in atherosclerosis. To explore the potential role of $\mathrm{HuR}$ in atherosclerosis, we generated smooth musclespecific HuR knockout mice and constructed an atherosclerotic model. As compared with controls, $\mathrm{HuR}^{\mathrm{SMKO}}$ mice more frequently exhibited atherosclerotic plaques and increased instability of plaques. Knockout of HuR inducing atherosclerosis was attributed to defective autophagy. Mechanically, HuR could bind to and stabilize the mRNAs of AMPK $\alpha 1$ and AMPK $\alpha 2$, thereby increasing the expression of $\mathrm{AMPK} \alpha$ and enhancing autophagy (Fig. 7F).

As a member of RNA-binding proteins, HuR has a primary role to bind to the target mRNAs and modulate their stability and translational efficiency ${ }^{28}$. HuR also interacts with other types of RNA, including small interfering RNA, long noncoding RNAs, and circular RNAs ${ }^{29}$. Binding to RNAs such as cyclinA, hypoxia-inducible factor-1, cyclooxygenase 2, B-cell lymphoma-2 is associated with proliferation and apoptosis; thus, $\mathrm{HuR}$ is related to the occurrence of tumorigenesis ${ }^{30,31}$. HuR regulates mRNAs of inflammatory factors such as interleukin 6 , so $\mathrm{HuR}$ may be involved in inflammatory diseases ${ }^{28,32}$. In addition, $\mathrm{HuR}$ is also involved in metabolic diseases such as diabetes by binding to glucose transporter (GLUT1) mRNA $^{33}$. After the discovery of the relationship between $\mathrm{HuR}$ and hypertension in VSMCs ${ }^{22}$, here we continued to use smooth muscle-specific HuR knockout mice to explore the role of $\mathrm{HuR}$ in atherosclerosis. Smoothmuscle $\mathrm{HuR}$ protected against the development of atherosclerosis by targeting AMPK $\alpha$, which expands our understanding of $\mathrm{HuR}$ in cardiovascular diseases.

AMPK is a serine/threonine-protein kinase that acts as a central component of the signaling pathway regulating the conversion between anabolism and catabolism ${ }^{34}$. AMPK is a heterotrimer composed of one catalytic subunit and two regulatory subunits. The AMPK subunits all have multiple isoforms. Differences in isoform composition affects AMPK localization and function. Catalytic subunit $\alpha$, which has two different isoforms, $\alpha 1$ and $\alpha 2$, is a major functional component of AMPK activation. Phosphorylation at Thr172 of $\alpha$ subunit is essential for

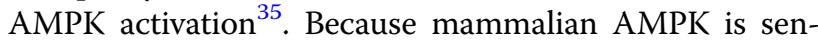
sitive to the AMP:ATP ratio, any cellular process that reduces ATP levels or increases AMP concentration could activate AMPK. Some cytokines such as leptin, adiponectin, and ghrelin and some drugs such as AICAR, A769662, and metformin can activate AMPK directly or indirectly $^{36}$. Also, AMPK activation is known to be mediated by liver kinase B1 (LKB1), $\mathrm{Ca}^{2+} /$ calmodulin- 
A

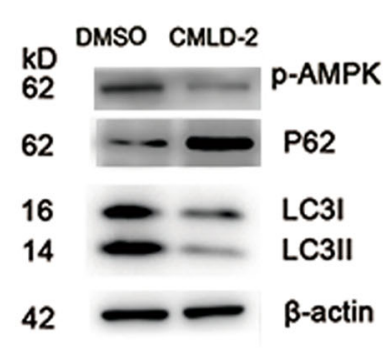

B

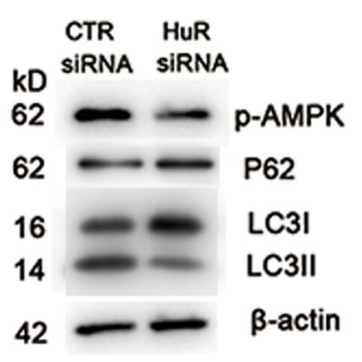

C

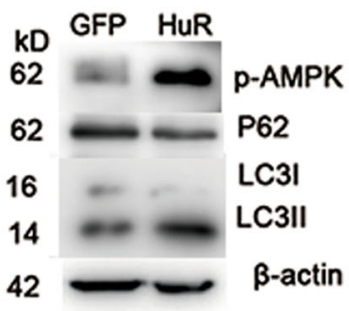

D

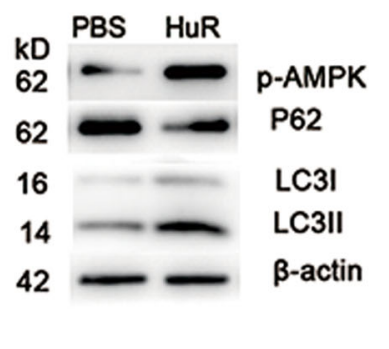

E

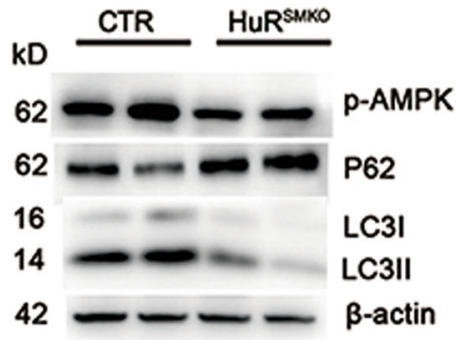

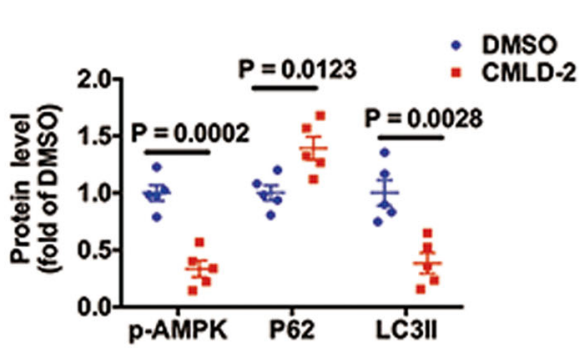
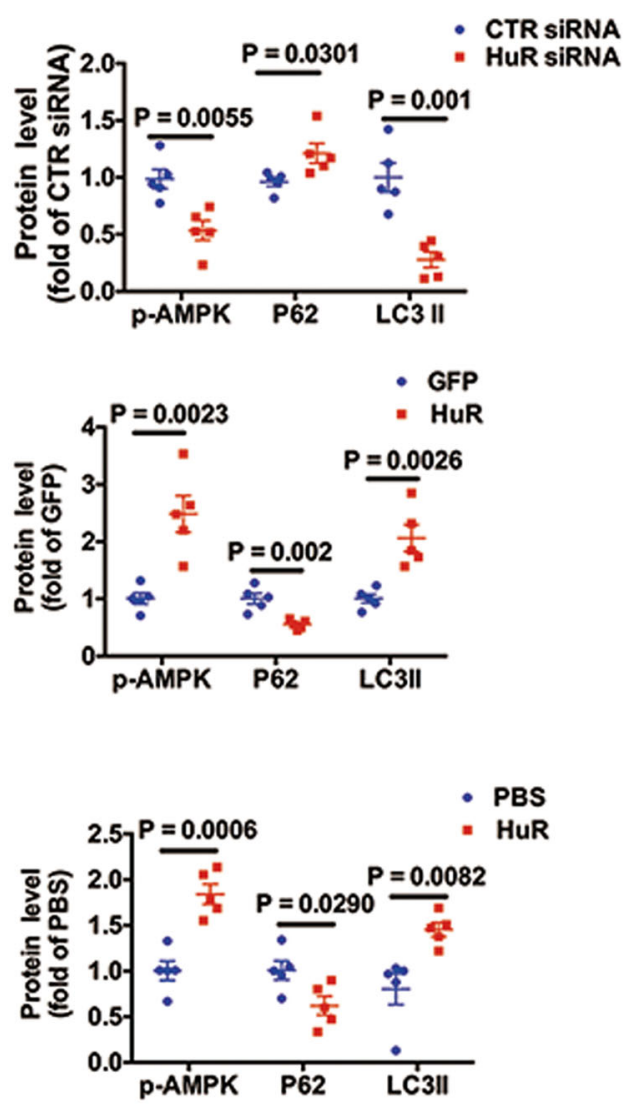

Fig. 6 HuR positively regulates autophagy. Western blot analysis of p-AMPK, p62, and LC3II in VSMCs. A Treated with DMSO or $30 \mu M$ CMLD-2 for $24 \mathrm{~h}(n=5)$. B Transfected with CTR or HuR siRNA for $48 \mathrm{~h}(n=5)$. C Infected with ad-GFP or ad-HuR $(n=5)$, and $\mathbf{D}$ treated with PBS or $0.5 \mu \mathrm{g} / \mu \mathrm{l}$ recombinant HuR protein for $48 \mathrm{~h}(n=5)$. E Western blot analysis of p-AMPK, p62, and LC3II in aortas from CTR and HuR ${ }^{\text {SMKO }}$ mice $(n=5)$. 
A
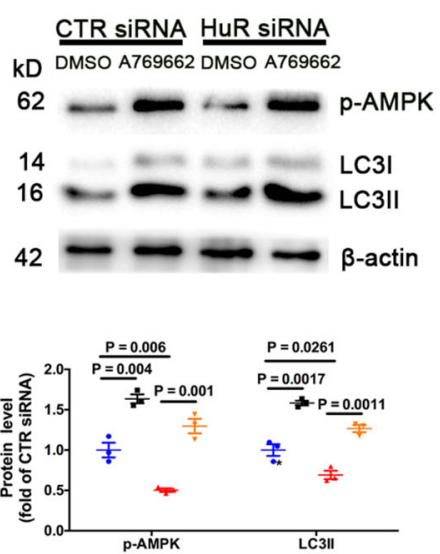

B
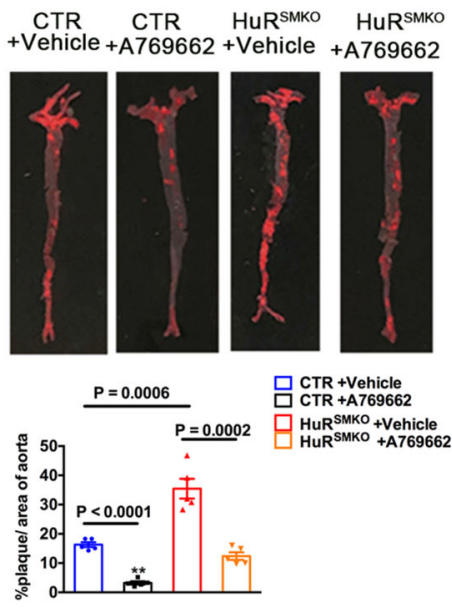

$\mathrm{E}$

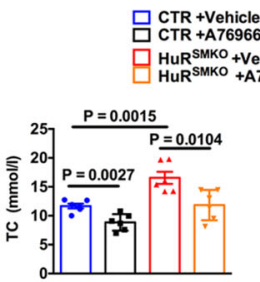

마 +Vehicle

HuR
HuR

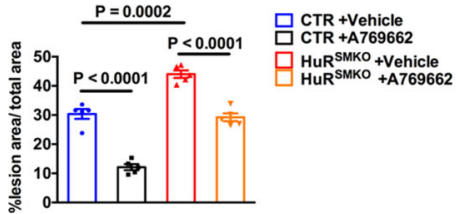

D

CTR+Vehicle HuR ${ }^{\mathrm{SMKO}}+$ Vehicle

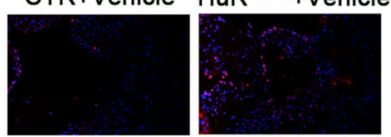

CTR+A769662 $\mathrm{HuR}^{\mathrm{SMKO}}+\mathrm{A} 769662$
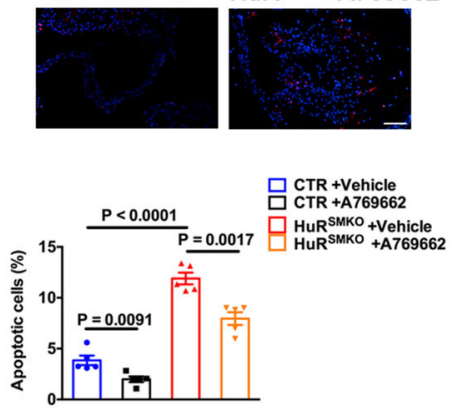

$\mathrm{F}$

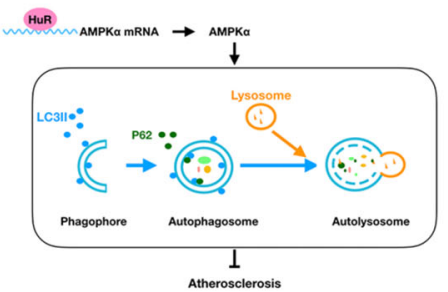

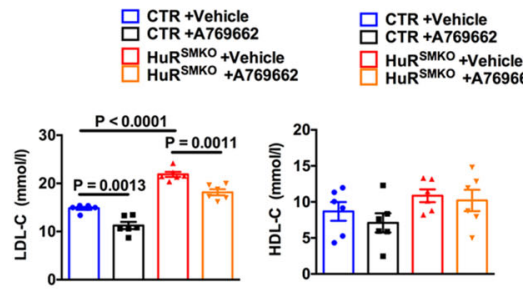

Fig. 7 Pharmacological AMPK activation induced autophagy and suppressed atherosclerosis in HuR ${ }^{\text {SMKO }}$ mice. A Western blot analysis of VSMCs transfected with CTR or HuR siRNA for $48 \mathrm{~h}$, then treated with $200 \mu \mathrm{M}$ A769662 for $1 \mathrm{~h}(n=5)$. CTR and HuR ${ }^{\text {SMKO }}$ mice were single injected with rAAV/D377Y-mPCSK9, then intraperitoneally injected with $30 \mathrm{mg} / \mathrm{kg}$ A769662 daily and fed a Paigen diet for 12 weeks. B Oil-red O staining in aortas $(n=5)$. C Oil-red O staining in aortic roots $(n=5)$. Scale bar $=200 \mu \mathrm{m}$. D TUNEL staining in aortic roots $(n=5)$. Scale bar $=50 \mu \mathrm{m}$. E TC, TG, HDL-C, and LDL-C levels $(n=6)$. F Diagram for the role of smooth-muscle HuR in atherosclerosis via AMPK-mediated autophagy. HuR indicates human antigen R; AMPK, Adenosine 5'-monophosphate-activated protein kinase. 
dependent kinase CaMKK2 (CaMKK $\beta)$, and transforming growth factor- $\beta$-activated kinase 1 (TAK1) $)^{24,36,37}$. Some studies have reported that $\Delta \mathrm{Np} 63 \alpha$, a p53 family member, and microRNAs such as mir-19 and mir-101 can regulate the expression of AMPK $\alpha^{38-40}$. Our study found that $\mathrm{HuR}$ could regulate AMPK $\alpha$, which enhances our understanding of the regulatory mechanisms of AMPK $\alpha$ expression and activation. Recent studies showed that AMPK plays an important role in lipid metabolism ${ }^{41,42}$. In this study, we demonstrated that AMPK $\alpha 1$ and AMPK $\alpha 2$ were the target genes of $\mathrm{HuR}$. Therefore, we speculated that HuR may regulate lipid metabolism through AMPK in hepatocytes. In the future study, hepatocyte-specific HuR knockout mice will be generated and these mice are predicted to have the phenotype with dyslipidemia. Whether and how HuR regulating the impact of AMPK on dyslipidemia will be explored.

Autophagy is a major intracellular degradation system that aims to dynamically cycle energy and matter for cell renewal and homeostasis ${ }^{43}$. The most important upstream regulators of autophagy are AMPK and mammalian target of rapamycin. Moreover, some studies have found that AMPK and mammalian target of rapamycin interact with each other ${ }^{44,45}$. From autophagosomes to autolysosomes, autophagy-related proteins such as Atg5, Atg7, LC3II, and p62 are indispensable for the occurrence and development of autophagy ${ }^{46}$. In recent years, the relationship between autophagy and atherosclerosis has also attracted much attention. Macrophage-specific Atg5 deficiency promotes atherosclerosis by interfering with cholesterol transport, apoptosis, and inflammation ${ }^{47-49}$. Endothelialspecific Atg5 and Atg7 deletion cause atherosclerotic plaque formation ${ }^{50,51}$. Knockout of Atg7 in VSMCs accelerates the process of atherosclerosis and promotes the regeneration of intima after injury ${ }^{15}$. Autophagy is closely related to cell senescence and apoptosis, which are also involved in atherosclerosis. In this study, knockout of HuR led to defective autophagy, which further increased apoptosis and atherosclerosis.

In summary, HuR could increase AMPK-mediated smooth muscle autophagy and play a protective role in atherosclerosis. Our research provides a new concept and drug target for treating atherosclerosis.

\section{Materials and methods Reagents}

Adenovirus expressing GFP, HuR (ad-HuR, ad-GFP), and recombinant adeno-associated viral of murine proprotein convertase subtilisin/kexin type 9 mutants (rAAV/D377Y-mPCSK9) were from Vigenebio (MD, USA). Adenovirus expressing LacZ (ad-LacZ) and GFPmRFP-LC3II (ad-GFP-mRFP-LC3II) were from Hanbio (Shanghai). Actinomycin $\mathrm{D}$ and Oil-red $\mathrm{O}$ were from Sigma (St Louis, MO, USA). A769662 was from Selleck
Chemical (Houston, TX, USA). Ox-LDL was from Yiyuan (Guangzhou, China). CMLD-2 was from Millipore (Temecula, CA, USA). Recombinant HuR protein was from Proteintech (Chicago, IL, USA). Control and HuR siRNA were synthesized by BioSune (Shanghai). The sequences for HuR siRNA were 5'-CCAGUUUCAAU GGUCAUAATT- $3^{\prime}$ and $5^{\prime}$-UUAUGACCAUUGAAACU GGTT-3' and control siRNA were 5'-GGUUGAAUCUG CAAAGCUUTT- $3^{\prime}$ and $5^{\prime}$-AAGCUUUGCAGAUUCAA CCTT-3'. Paigen diet was from Trophic Diets (TP28640, China), containing $15 \%$ fat, $0.5 \%$ bile salt, and $1.25 \%$ cholesterol.

\section{Cell culture and infection}

Mouse smooth muscle cells were from ATCC (Manassas, VA, USA) with STR authentication. Cells were cultured in DMEM containing 10\% fetal bovine serum and $50 \mu \mathrm{g} / \mathrm{ml}$ penicillin/streptomycin and seeded in 6-well plates at $1.0 \times 10^{4}$ cells $/ \mathrm{cm}^{2}$. VSMCs grown to $70 \%$ confluence were infected with adenovirus at multiplicity of infection 75 for $48 \mathrm{~h}$.

\section{Mouse models}

Smooth muscle-specific HuR knockout $\left(\mathrm{HuR}^{\mathrm{SMKO}}\right)$ mice were generated as described ${ }^{22}$. Male control and $\mathrm{HuR}^{\mathrm{SMKO}}$ mice at 8 weeks old were given a single tail-vein injection with $\mathrm{rAAV} / \mathrm{D} 377 \mathrm{Y}-\mathrm{mPCSK} 9$ at $1.5 \times 10^{11} \mathrm{pfu}$ for each mouse as described ${ }^{52}$ and fed a Paigen diet for 12 weeks. Male apolipoprotein E-deficient mice $\left(\mathrm{ApoE}^{-1-}\right)$ at 8 weeks old were from Vital River (Beijing) and were divided into two groups for high-fat diet (HFD) or normal chow diet (ND) feeding. Mice were housed at $25^{\circ} \mathrm{C}, 12$-h light/dark. Mice were euthanized using profound anesthesia with $4 \%$ isoflurane followed by exsanguination and tissue removal. The animal experiment was approved by the Animal Care Committee of Shandong University and was performed in compliance with the Animal Management Rules of the Chinese Ministry of Health. All animal experiments were performed conform the guidelines from Directive 2010/63/EU of the European Parliament on the protection of animals used for scientific purposes.

\section{Atherosclerotic lesion assay}

Mice were euthanized and perfused with PBS. Hearts and aortas from the proximal ascending aorta to the abdominal aorta were removed and fixed in $4 \%$ paraformaldehyde. Aortas were dissected free of fat and adventitial tissue, opened longitudinally, stained with $0.5 \%$ freshly-made Oilred $\mathrm{O}$ for $2 \mathrm{~h}$, and pinned onto black silicon plates for imaging. After optical cutting temperature (OCT) compound embedding, the heart was cut to the aortic root to reveal the valvular lobe, stained with $0.5 \%$ Oil-red $\mathrm{O}$ and observed by microscopy. The results were reported as percentage of lesion area to total aortic area. 


\section{Morphology of aorta and aortic root}

Aortas and hearts were embedded in OCT and sliced into 5 - $\mu \mathrm{m}$-thick frozen sections. The heart was cut to the aortic root. After sections were hydrated, immunofluorescence, immunohistochemistry, or other special staining was performed. To detect HuR-knockout efficiency in VSMCs, fluorescent double labeling of aortic sections was performed with HuR antibody (1:300, Millipore) and $\alpha$-SMA antibody (1:300, Abcam). Sections of aortic roots were immunostained with MOMA-2 antibody (1:300, Abcam) to detect macrophage content and $\alpha$-SMA antibody to detect VSMC content. The collagen of aortic root was detected by using a Masson staining kit (Solarbio, Beijing). The plaque vulnerability index was calculated as follows: (macrophage staining \%+lipid staining $\%) /(\text { SMC staining } \%+\text { collagen staining } \%)^{53}$.

\section{Detection of apoptosis}

Apoptosis was detected by immunohistochemical staining with cleaved caspase-3 antibody (1:300, Affinity) and terminal UTP nick end-labeling (TUNEL). Briefly, aortic root sections were fixed in fresh $4 \%$ paraformaldehyde for $20 \mathrm{~min}$ at $15-25^{\circ} \mathrm{C}$, then incubated with premeabilization solution containing $0.1 \%$ Triton X-100 and $0.1 \%$ sodium citrates for $2 \mathrm{~min}$ at $2-8{ }^{\circ} \mathrm{C}$. Staining followed the recommendations of the in-situ Cell Death Detection Kit (Roche, Basel, Switzerland).

\section{Lipid profile assays}

Serum was isolated for determining levels of total cholesterol (TC), triglycerides (TG), high-density lipoprotein cholesterol (HDL-C), and low-density lipoprotein cholesterol (LDL-C). The blood lipid assay kit was from Jiancheng Bioengineering Institute (Nanjing, China).

\section{Western blot analysis}

A 15- $\mu$ g amount of protein lysates from isolated aorta and SMCs were run on a 12\% SDS-PAGE gel and immunoblotted overnight with the primary antibodies for HuR $(29 \mathrm{ng} / \mathrm{ml}, 12582 \mathrm{~S}, \mathrm{CST})$, AMPK $\alpha 1 \quad(1 \mu \mathrm{g} / \mathrm{ml}$,

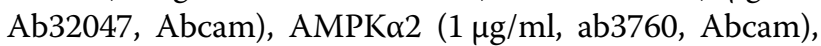
p-AMPK (27 ng/ml, 2535S, CST), p62 (293 ng/ml, 184201-AP, Proteintech), LC3I/II $(64.5 \mathrm{ng} / \mathrm{ml}, 4108 \mathrm{~S}, \mathrm{CST})$, Cleaved Caspase-3 $(1 \mu \mathrm{g} / \mathrm{ml}$, BF0711, Affinity), and $\beta$-actin $(1 \mu \mathrm{g} / \mathrm{ml}, 20536-1-A P$, Proteintech).

\section{RNA immunoprecipitation assay}

Whole-cell lysates were incubated overnight at $4{ }^{\circ} \mathrm{C}$ with protein $\mathrm{A} / \mathrm{G}$ beads pre-conjugated with $5 \mu \mathrm{g}$ rabbit IgG or $\mathrm{HuR}$ antibody (Millipore). RNA was then isolated from immunoprecipitates by using the Magna RIP kit (Millipore) with the primer sequences AMPKo1: 5'-GGC ACCTTCGGGAAAGTGAA- $3^{\prime}$ and $5^{\prime}$-TGTGAGGGTG CCTGAACAGC-3' and AMPK $\alpha 2: 5^{\prime}$-GAAGATTCGCA
GTTTAGATGTTG- $3^{\prime}$ and $5^{\prime}$-TCGAACAATTCACCTC CAGA-3'.

\section{GFP-mRFP-LC3II punctation}

VSMCs were infected with ad-GFP-mRFP-LC3II for $48 \mathrm{~h}$. LC3II spots were observed by fluorescence microscopy (Nikon, Tokyo). GFP-RFP-LC3II serves as a specific marker for autophagic flux that relies on the different nature of GFP and RFP fluorescence under acidic conditions. Because GFP was quenched in the lysosomal acidic conditions, autophagosomes are shown as yellow puncta $\left(\mathrm{RFP}^{+} \mathrm{GFP}^{+}\right)$, and autolysosomes are shown as red puncta $\left(\mathrm{RFP}^{+} \mathrm{GFP}^{-}\right)$in green- and red-merged images ${ }^{54}$. The mean number of LC3II points per cell was calculated from more than 30 cells.

\section{Transmission electron microscopy}

After infection with ad-LacZ or HuR for $48 \mathrm{~h}$, VSMCs were harvested and fixed in electron microscope fixative containing $2.5 \%$ glutaraldehyde (Servicebio, Wuhan, China) for $2 \mathrm{~h}$. Samples were fixed in $1 \%$ osmium tetroxide and dehydrated with ethanol in ascending order, followed by epoxide resin-embedding and cut into sections. Images of stained sections were collected by transmission electron microscopy (HT7700, Hitachi, Japan).

\section{Statistical analysis}

Group allocation for the experiments was randomized and not blinded. Sample analyses were not blinded. Data are expressed as mean \pm SEM and were analyzed by using GraphPad Prism 6.0. All data were tested for normal distribution and equal variances. Student $t$ test was used to compare two groups with $95 \%$ confidence interval. One-way ANOVA and Bonferroni post-tests were used in four groups. Statistical significance was set at $P<0.05$.

\section{Acknowledgements}

The authors thank Prof Xiao Yang (State Key Laboratory of Proteomics, Collaborative Innovation Center for Cardiovascular Disorders, Genetic Laboratory of Development and Diseases, Institute of Biotechnology, Beijing, PR China) for providing the a-SMA-Cre mice for this study.

\footnotetext{
Author details

${ }^{1}$ The Key Laboratory of Cardiovascular Remodeling and Function Research, Chinese Ministry of Education, Chinese National Health Commission and Chinese Academy of Medical Sciences, The State and Shandong Province Joint Key Laboratory of Translational Cardiovascular Medicine, Department of Cardiology, Qilu Hospital, Cheeloo College of Medicine, Shandong University, Jinan, China. ${ }^{2}$ Cardiovascular Disease Research Center of Shandong First Medical University, Central Hospital Affiliated to Shandong First Medical University, Jinan, China. ${ }^{3}$ Department of General Surgery, Qilu Hospital of Shandong University, Jinan, China. ${ }^{4}$ Department of Physiology and Pathophysiology, School of Basic Medical Sciences, Shandong University, Jinan, China. ${ }^{5}$ Department of Histology and Embryology, School of Basic Medical Sciences, Shandong University, Jinan, China
}

\section{Author contributions}

S.L. designed and performed the research, analyzed data, and wrote the manuscript. X.J., X.C., and J.W. performed the research. S.L. and H.L. analyzed 
data. C.Z. and J.Y. reviewed data, W.Z. designed the research, reviewed data, and wrote the manuscript.

\section{Funding}

This study was supported by grants from the National Natural Science Foundation of China (no. 81970198, 81770473) and the Taishan Scholar Project of Shandong Province of China (no. tsqn20161066), the Natural Science Foundation for Distinguished Young Scholars of Shandong Province (ZR2020JQ30), and the Natural Science Foundation of Shandong Province (no. ZR2020MH132).

\section{Conflict of interest}

The authors declare no competing interests.

\section{Ethics statement}

The animal experiment was approved by the Animal Care Committee of Shandong University and was performed in compliance with the Animal Management Rules of the Chinese Ministry of Health. All animal experiments were performed conform the guidelines from Directive 2010/63/EU of the European Parliament on the protection of animals used for scientific purposes.

\section{Publisher's note}

Springer Nature remains neutral with regard to jurisdictional claims in published maps and institutional affiliations.

Supplementary information The online version contains supplementary material available at https://doi.org/10.1038/s41419-021-03671-2.

Received: 2 November 2020 Revised: 19 March 2021 Accepted: 23 March 2021

Published online: 09 April 2021

\section{References}

1. Meyer, M. R. et al. G protein-coupled estrogen receptor protects from atherosclerosis. Sci. Rep. 4, 7564 (2014).

2. Gimbrone, M. A. Jr \& Garcia-Cardena, G. Endothelial cell dysfunction and the pathobiology of atherosclerosis. Circ. Res. 118, 620-636 (2016).

3. Lee, Y. T. et al. Mouse models of atherosclerosis: a historical perspective and recent advances. Lipids Health Dis. 16, 12 (2017).

4. Libby, P., Ridker, P. M. \& Hansson, G. K. Progress and challenges in translating the biology of atherosclerosis. Nature 473, 317-325 (2011).

5. Li, B., Xia, Y. \& Hu, B. Infection and atherosclerosis: TLR-dependent pathways. Cell Mol. Life Sci. 77, 2751-2769 (2020).

6. Allahverdian, S., Chehroudi, A. C., McManus, B. M., Abraham, T. \& Francis, G. A. Contribution of intimal smooth muscle cells to cholesterol accumulation and macrophage-like cells in human atherosclerosis. Circulation 129, 1551-1559 (2014).

7. Bennett, M. R., Sinha, S. \& Owens, G. K. Vascular smooth muscle cells in atherosclerosis. Circ. Res. 118, 692-702 (2016).

8. Lim, S. \& Park, S. Role of vascular smooth muscle cell in the inflammation of atherosclerosis. BMB Rep. 47, 1-7 (2014).

9. Grootaert, M. O. J. et al. Vascular smooth muscle cell death, autophagy and senescence in atherosclerosis. Cardiovasc Res. 114, 622-634 (2018).

10. Kuma, A., Komatsu, M. \& Mizushima, N. Autophagy-monitoring and autophagy-deficient mice. Autophagy 13, 1619-1628 (2017).

11. Mei, Y., Thompson, M. D., Cohen, R. A. \& Tong, X. Autophagy and oxidative stress in cardiovascular diseases. Biochim Biophys. Acta 1852, 243-251 (2015).

12. Mondaca-Ruff, D. et al. Angiotensin II-regulated autophagy is required for vascular smooth muscle cell hypertrophy. Front Pharm. 9, 1553 (2019).

13. Ouimet, M. et al. MicroRNA-33 regulates macrophage autophagy in atherosclerosis. Arterioscler Thromb. Vasc. Biol. 37, 1058-1067 (2017).

14. Osonoi, Y. et al. Defective autophagy in vascular smooth muscle cells enhances cell death and atherosclerosis. Autophagy 14, 1991-2006 (2018).

15. Grootaert, M. O. et al. Defective autophagy in vascular smooth muscle cells accelerates senescence and promotes neointima formation and atherogenesis. Autophagy 11, 2014-2032 (2015).

16. Lal, S. et al. CRISPR knockout of the HuR gene causes a xenograft lethal phenotype. Mol. Cancer Res. 15, 696-707 (2017).
17. Wang, W. et al. AMP-activated protein kinase-regulated phosphorylation and acetylation of importin alpha1: Involvement in the nuclear import of RNAbinding protein HuR. J. Biol. Chem. 279, 48376-48388 (2004).

18. Grammatikakis, I., Abdelmohsen, K \& Gorospe, M. Posttranslational control of HuR function. Wiley Interdiscip. Rev. RNA 8, https:/doi.org/10.1002/wrna.1372 (2017).

19. Wang, J. et al. Multiple functions of the RNA-binding protein HuR in cancer progression, treatment responses and prognosis. Int. J. Mol. Sci. 14, 10015-10041 (2013).

20. Sun, K et al. Neuron-specific HuR-deficient mice spontaneously develop motor neuron disease. J. Immunol. 201, 157-166 (2018).

21. Li, J. et al. Adipose HuR protects against diet-induced obesity and insulin resistance. Nat. Commun. 10, 2375 (2019).

22. Liu, S. et al. HuR (human antigen R) regulates the contraction of vascular smooth muscle and maintains blood pressure. Arterioscler Thromb. Vasc. Biol. 40, 943-957 (2020).

23. Grootaert, M. O. J., Roth, L., Schrijvers, D. M., De Meyer, G. R. Y. \& Martinet, W. Defective autophagy in atherosclerosis: to die or to senesce? Oxid. Med. Cell Longev. 2018, 7687083 (2018).

24. Tamargo-Gomez, I. \& Marino, G. AMPK: regulation of metabolic dynamics in the context of autophagy. Int. J. Mol. Sci. 19, 3812 (2018).

25. Kim, J., Kundu, M., Viollet, B. \& Guan, K. L. AMPK and mTOR regulate autophagy through direct phosphorylation of Ulk1. Nat. Cell Biol. 13, 132-141 (2011).

26. Pullmann, R. Jr et al. Enhanced proliferation of cultured human vascular smooth muscle cells linked to increased function of RNA-binding protein HuR J. Biol. Chem. 17, 22819-22826 (2005).

27. Ray, M. et al. Genetic deletion of IL-19 (interleukin-19) exacerbates atherogenesis in $1119^{-1-} \times \mathrm{Ldlr}^{-1-}$ double knockout mice by dysregulation of mRNA stability protein HuR (human antigen R). Arterioscler Thromb. Vasc. Biol. 38, 1297-1308 (2018)

28. Ouhara, K et al. The induced rna-binding protein, HuR, targets 3 '-UTR region of IL-6 mRNA and enhances its stabilization in periodontitis. Clin. Exp. Immunol. 192, 325-336 (2018).

29. Abdelmohsen, $\mathrm{K}$. et al. Identification of HuR target circular RNAs uncovers suppression of PABPN1 translation by CircPABPN1. RNA Biol. 14, 361-369 (2017).

30. Liu, Y. et al. The Jun/miR-22/HuR regulatory axis contributes to tumourigenesis in colorectal cancer. Mol. Cancer 17, 11 (2018).

31. Eberhardt, W., Nasrullah, U. \& Haeussler, K. Inhibition of caspase-2 translation by the mRNA binding protein HuR: a novel path of therapy resistance in colon carcinoma cells? Cells 8, 797 (2019).

32. Christodoulou-Vafeiadou, E. et al. Divergent innate and epithelial functions of the RNA-binding protein HuR in intestinal inflammation. Front. Immunol. 9 2732 (2018).

33. Gantt, K. R. et al. The regulation of glucose transporter (GLUT1) expression by the RNA binding protein HuR. J. Cell Biochem. 99, 565-574 (2016)

34. Pulinilkunnil, T. et al. Adrenergic regulation of AMP-activated protein kinase in brown adipose tissuein vivo. J. Biol. Chem. 286, 8798-8809 (2011).

35. Hardman, S. E., Hall, D. E., Cabrera, A. J., Hancock, C. R. \& Thomson, D. M. The effects of age and muscle contraction on AMPK activity and heterotrimer composition. Exp. Gerontol. 55, 120-128 (2014).

36. Shirwany, N. A. \& Zou, M.-H. AMPK in cardiovascular health and disease. Acta Pharmacologica Sin. 31, 1075-1084 (2010).

37. Salminen, A. \& Kaarniranta, K. AMP-activated protein kinase (AMPK) controls the aging process via an integrated signaling network. Ageing Res. Rev. 11 230-241 (2012).

38. Mavrakis, K. J. et al. Genome-wide RNA-mediated interference screen identifies miR-19 targets in Notch-induced T-cell acute lymphoblastic leukaemia. Nat. Cell Biol. 12, 372-379 (2010).

39. Liu, P. et al. Mir-101-3p is a key regulator of tumor metabolism in triple negative breast cancer targeting AMPK. Oncotarget 7, 35188-35198 (2016).

40. Yi, Y. et al. Transcriptional suppression of AMPKa1 promotes breast cancer metastasis upon oncogene activation. Proc. Natl Acad. Sci. USA 117 8013-8021 (2020).

41. Sun, S. M. et al. AMPK activator C24 inhibits hepatic lipogenesis and ameliorates dyslipidemia in HFHC diet-induced animal models. Acta Pharm. Sin. https://doi.org/10.1038/s41401-020-0472-9 (2020).

42. Sun, L. et al. Hydrogen sulfide reduces serum triglyceride by activating liver autophagy via the AMPK-mTOR pathway. Am. J. Physiol. Endocrinol. Metab. 309, E925-E935 (2015).

43. Mizushima, N. \& Komatsu, M. Autophagy: renovation of cells and tissues. Cell 147, 728-741 (2011). 
44. Alloza, l., Goikuria, H., Freijo, M. D. M. \& Vandenbroeck, K. A role for autophagy in carotid atherosclerosis. Eur. Stroke J. 1, 255-263 (2016).

45. Holczer, M. et al. A double negative feedback loop between mTORC1 and AMPK kinases guarantees precise autophagy induction upon cellular stress. Int. J. Mol. Sci. 20, 5543 (2019).

46. De Meyer, G. R. et al. Autophagy in vascular disease. Circ. Res. 116, 468-479 (2015).

47. Liao, $X$. et al. Macrophage autophagy plays a protective role in advanced atherosclerosis. Cell Metab. 15, 545-553 (2012).

48. Razani, B. et al. Autophagy links inflammasomes to atherosclerotic progression. Cell Metab. 15, 534-544 (2012).

49. Liu, K. et al. Impaired macrophage autophagy increases the immune response in obese mice by promoting proinflammatory macrophage polarization. Autophagy 11, 271-284 (2015).
50. Torisu, K. S. K. et al. Intact endothelial autophagy is required to maintain vascular lipid homeostasis. Aging Cell 15, 187-191 (2016)

51. Vion, A. C. et al. Autophagy is required for endothelial cell alignment and atheroprotection under physiological blood flow. Proc. Natl Acad. Sci. USA 114 E8675-E8684 (2017)

52. Bjørklund, M. M. et al. Induction of atherosclerosis in mice and hamsters without germline genetic engineering. Circ. Res. 114, 1684-1689 (2014).

53. $\mathrm{Li}, \mathrm{H}$. et al. Melatonin enhances atherosclerotic plaque stability by inducing prolyl-4-hydroxylase a1 expression. J. Hypertens. 37, 964-971 (2019).

54. Zhang, Y. et al. Mir143-BBC3 cascade reduces microglial survival via interplay between apoptosis and autophagy: implications for methamphetaminemediated neurotoxicity. Autophagy 12, 1538-1559 (2016). 